\title{
Evaluating Subgrid-Scale Models for Large-Eddy Simulation of Turbulent Katabatic Flow
}

\author{
Bryan A. Burkholder, Evgeni Fedorovich, and Alan Shapiro
}

School of Meteorology, University of Oklahoma, 120 David L. Boren Blvd., Norman, OK, USA 73072 Bryan.A.Burkholder-1@ou.edu

\begin{abstract}
Summary. The performance of commonly used subgrid-scale (SGS) models is evaluated for large-eddy simulation (LES) of turbulent katabatic flow. The very stable stratification and strong low-level shear in this flow provide a stringent test for SGS models. Using an a posteriori testing procedure, the SGS models' performance in reproducing turbulence statistics and spectra in katabatic flow is investigated.
\end{abstract}

Key words: Large-Eddy Simulation, Subgrid-Scale Models, Katabatic Flow, A Posteriori Testing

\section{Introduction}

Over the past few decades, large-eddy simulation (LES) has become an invaluable tool for investigating the structure and characteristics of atmospheric boundary layer flows [1]. While encouraging results have been obtained from LES of neutrally stratified and unstable (convective) boundary layers, questions still remain concerning the reliability of LES for reproducing stably-stratified turbulent boundary layers [2]. Under stably-stratified conditions, the characteristic length scale of the small-scale turbulent motions decrease, placing a larger burden on the subgrid-scale model employed.

A plethora of subgrid/subfilter scale (SGS/SFS) models exist for wide variety of applied problems in atmospheric dynamics. Reliable simulations have been performed in moderately stable boundary layers and have been successfully compared to observational data [3]. However, no single SGS model appears significantly more appropriate than another for a broad range of grid spacings. Typically, intercomparisons test LES performance against direct numerical simulation (DNS) or measurement data. However, LES is run at much coarser resolutions than DNS and outputs from the two simulation techniques may not be directly comparable. Similarly, LES may not incorporate all of the phenomena present in real flows when compared to observational data. To fairly evaluate the performance of the SGS models, we employ an a posteriori testing procedure [4][5]. To perform this type of procedure, the 
statistical behavior of the flow reproduced by LES is compared to filtered DNS data for an identically forced flow.

We employ the a posteriori testing procedure in simulations of a shallow jet-like flow developing along a cooled planar slope (katabatic flow). The earliest solutions to this flow type can be traced back to studies by Prandtl [6], where the Boussinesq equations of motion were solved analytically for a laminar slope flow in a stablystratified environment. The Prandtl solution is characterized by a strong near-surface jet and weaker upslope flow aloft. It is no surprise that the turbulent counterpart of this flow is particularly difficult for LES, given the strong flow shear near the surface accompanied by strong stable stratification. To futher complicate matters, no complete similarity theory has been developed yet for flows along sloping terrain [7]. These factors only compound the problems LES is known to have near bounding surfaces, where the characteristic length scales of turbulent motions can be close to the LES filter width [8]. Katabatic and anabatic (heated slope) flows have actually been numerically investigated using LES with two different SGS closures [9][10], but the question regarding the optimal closure of LES for katabatic flows remains unresolved.

\section{Governing Equations and Closures}

Following [6][11], we simulate a katabatic flow over a doubly-inifinite sloping surface which is inclined at a constant angle $\alpha$ with respect to the horizontal. In DNS, we solve the Boussinesq equations of motion and thermodynamic energy in rotated coordinates analagous to the ones adopted in [12][13] (Fig. 1). In the rotated coordinate system, $x=x_{1}$ points in the downslope direction, $y=x_{2}$ lies in the cross-slope direction, and $z=x_{3}$ is oriented normal to the slope.

In LES, we use the same rotated coordinates and solve the filtered Boussinesq equations of motion and thermodynamic energy,

$$
\frac{\partial \tilde{u}_{i}}{\partial t}+\tilde{u_{j}} \frac{\partial \tilde{u}_{i}}{\partial x_{j}}=\tilde{b}\left(-\delta_{i 1} \sin \alpha+\delta_{i 3} \cos \alpha\right)+v \frac{\partial \tilde{u}_{i}}{\partial x_{j} \partial x_{j}}-\frac{\partial \tau_{i j}}{\partial x_{j}}-\frac{1}{\rho} \frac{\partial \overline{\tilde{p}}}{\partial x_{i}},
$$
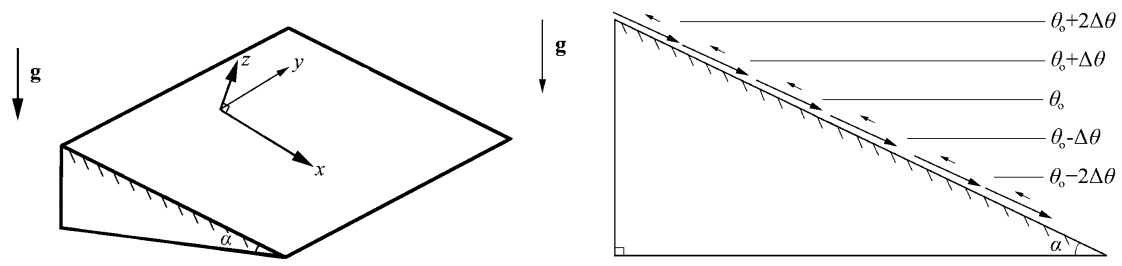

Fig. 1. Orientation of the coordinate system used (left) and a cross-sectional view of an idealized katabatic flow (right). The thin solid lines illustrate the stratification of the base-state environment $\left(\theta_{\infty}\right)$, which is characterized by $\theta$ increasing linearly with height. 


$$
\begin{gathered}
\frac{\partial \tilde{b}}{\partial t}+\tilde{u}_{j} \frac{\partial \tilde{b}}{\partial x_{j}}=-N^{2}\left(-\tilde{u}_{1} \sin \alpha+\tilde{u}_{3} \cos \alpha\right)+\kappa \frac{\partial^{2} \tilde{b}}{\partial x_{j} \partial x_{j}}-\frac{\partial B_{j}}{\partial x_{j}}, \\
\frac{\partial \tilde{u}_{i}}{\partial x_{i}}=0
\end{gathered}
$$

with

$$
\begin{aligned}
\tau_{i j} & =\widetilde{u_{i} u_{j}}-\tilde{u_{i}} \tilde{u_{j}}, \\
B_{j} & =\widetilde{b u_{j}}-\tilde{b} \tilde{u_{j}}, \\
\overline{\tilde{p}} & =\tilde{p}+\frac{2}{3} E,
\end{aligned}
$$

where $\widetilde{(\ldots)}$ indicates a quantity filtered using a top-hat filter, $N$ is the Brunt-Vaisala frequency (which is set to a constant value), $\tau_{i j}$ is the subgrid momentum flux (negative of the subgrid stress tensor), $B_{j}$ is the subgrid buoyancy flux, $\tilde{p}$ is the filtered pressure, $\overline{\tilde{p}}$ is the modified pressure, and $E$ is the subgrid/subfilter turbulence kinetic energy (TKE). The top-hat filter width, $\Delta$, is taken to be equal to the LES grid spacing. Buoyancy is defined as

$$
b=g \frac{\theta-\theta_{\infty}}{\theta_{r}},
$$

where $g$ is gravitational acceleration, $\theta$ is potential temperature, $\theta_{r}$ is a constant reference potential temperature, and $\theta_{\infty}$ is the environmental potential temperature which is taken to vary linearly with height. To close the system of filtered equations, $\tau_{i j}$ and $B_{j}$ must be parameterized in terms of the resolved flow fields.

One of the earliest SGS models used for meteorological applications was the Smagorinsky model [14], which employs the assumption of a balance between shear production and the dissipation of subgrid TKE. As a result, the SGS stress tensor is taken proportional to the resolved strain rate tensor:

$$
\begin{gathered}
v_{T}=\left[C_{S} \Delta\right]^{2} \tilde{S}, \\
\tau_{i j}=E \delta_{i j}-2 v_{T} \tilde{S}_{i j},
\end{gathered}
$$

where $\tilde{S}=\sqrt{2 \tilde{S}_{i j} \tilde{S}_{i j}}$ and $C_{S}$ is known as the Smagorinsky coefficient. Using a sharp cutoff filter in the inertial subrange and assuming the Kolmogorov scaling, $C_{S}$ was found to be roughly 0.17 [15]. The value of $C_{S}$ has also been found to vary significantly depending on the type of flow simulated [8]. A value around 0.2 is commonly used in atmospheric contexts [1]. While reasonable results can be attained using the Smagorinsky model, the underlying assumption that the SGS stress tensor is proportional to the strain rate is a critical one, and may not necessarily be valid in all applications. The model is also known to be overly dissipative, especially near the surface. Despite its inherent disadvantages, this SGS model is still implemented as a baseline model in LES of many atmospheric flows because of its simplicity.

Deardorff [16] considered another form of the subgrid TKE balance by including buoyancy effects and subgrid energy transport. The subgrid TKE is then calculated 
as a prognostic variable from a simplified version of its governing equation [17] and is used to parameterize the eddy viscosity locally through

$$
\begin{gathered}
v_{T}=C_{D} l \sqrt{E}, \\
\kappa_{T}=\left(1+\frac{2 l}{\Delta}\right) v_{T}, \\
l= \begin{cases}\Delta & \frac{\partial \tilde{b}}{\partial z} \leq 0, \\
\min [\Delta, 0.5 \sqrt{E /(\partial \tilde{b} / \partial z)}] & \frac{\partial \tilde{b}}{\partial z}>0 .\end{cases}
\end{gathered}
$$

The adopted dependence of the mixing length on the stratification (Eq. 12) is used to decrease the turbulent length scale when stable stratification is encountered. The parameter $C_{D}$ was set to 0.12 in [16]. Even though [16] used this $l$ scaling factor within stratocumulus cloud layers, it is currently implemented in many SGS models that are used in general atmospheric applications.

Both considered formulations, however, do not account for the backscatter of energy from small scales to larger scales, which may be important in parameterizing the SGS motions [18][19][20]. To counteract the overly dissipative nature of the schemes proposed by [14] and [16], some form of non-linear or stochastic backscattering mechanism has been suggested for simulations of stable boundary layers in [1][21]. The original stochastic backscattering model of [19] was computationally exhaustive and did not match Monin-Obukhov similarity near the surface. Sullivan [22] argued that this backscatter behavior could be accounted for by using adequate grid resolution and less dissipative SGS closures. A two-part model was proposed, in which the near-surface region was controlled by the mean shear and the region away from the surface followed the closure [16]:

$$
\tau_{i j}=-2 v_{T}^{\prime} \gamma \tilde{S}_{i j}-2 \bar{v}_{T}\left\langle\tilde{S}_{i j}\right\rangle+E \delta_{i j}
$$

where $\langle\ldots\rangle$ represents averaging in homogeneous directions (slope-parallel planes in the case of laterally homogeneous flow), $v_{T}{ }^{\prime}$ is the fluctuating field-eddy viscosity, $\bar{v}_{T}$ is the mean-field eddy viscosity, and $\gamma$ is the isotropy factor:

$$
\gamma=\frac{\tilde{S}^{\prime}}{\langle\tilde{S}\rangle+\tilde{S}^{\prime}},
$$

where $\tilde{S}^{\prime}$ is the fluctuating strain rate. The calculation of the isotropy factor allows a smooth transition from an ensemble-average approach near the ground to the baseline subgrid TKE model of [16] in the main portion of the flow. The SGS buoyancy flux was found to be important in moderately stable conditions and was later included in the two-part formulation [23].

Another method to modify the classic Smagorinsky model is to dynamically calculate $C_{S}$. This approach employs a larger filter width, for example, $\widehat{\Delta}=2 \Delta$, and 
then uses the filtered fields to calculate the Smagorinsky coefficient using the Germano identity [24]. Rather than specifying the coefficient, the SGS model essentially calculates its optimal value from the resolved fields. To statistically minimize the error and find this optimal value, averaging over some homogeneous direction must be applied [25]. Preferably, this averaging should be performed over Lagrangian paths or over the nearest grid cell neighbors in homogeneous directions [26]. A third, even larger filter width, $\tilde{\Delta}=2 \widehat{\Delta}=4 \Delta$, can be introduced to make the model scaledependent using a power-law relation between filter widths [27][28]. Even though the dynamic versions of the original Smagorinsky type-closure can essentially adjust the characteristic length scale of the subgrid-scale motions, it cannot reproduce the backscattering and anisotropy necessary to correctly simulate the turbulence in the stable boundary layer [21].

\section{Model setup and flow characteristics}

The DNS code used in our study is described in [11]. It uses second-order in space finite difference approximations of spatial derivatives and a leapfrog scheme with a weak Asselin filter for time advancement. The modified pressure is computed diagnostically at every time step by a Poisson solver. The LES uses the same core of the computational code, but employs various SGS algorithms for solving the filtered Boussinesq equations. Table 1 lists the different subgrid closures that have been incorporated in the LES and tested in the present study. The naming convention adopted for the various SGS closures is as follows: Smagorinsky (S63) [14], the two-part model (S94) [22], and the scale-invariant dynamic model (PA00) [27][28]. In the case of the Deardorff [16] closure, two versions are employed; one using the $l$-scaling factor given in Eq. 12 (D80) and another that takes $\Delta$ to be the mixing length regardless of the stratification [D80 (no N)]. The LES with no SGS closure, commonly referred to as a quasi-DNS, is abbreviated as qDNS. For the filtered DNS output, the abbreviation is of fDNS is taken. The LES runs are carried out with grid sizes of $32 \times 32 \times 100$, while the DNS is run on the grid $128 \times 128 \times 400$. This gives the LES a degraded resolution by a factor of four. The filtering procedures used in the a posteriori test are described in Sect. 4.

To generate the katabatic flow in the simulations, a buoyancy flux of $-0.5 \mathrm{~m}^{2} \mathrm{~s}^{-3}$ is imposed at the surface. The boundary conditions for the DNS are taken to be noslip and impermeable at the surface and zero-gradient for all variables at the top of the domain. The Prandtl number, $\operatorname{Pr}=v / \kappa$, is taken to be unity. In the LES, MoninObukhov similarity is applied to formulate surface boundary conditions in the runs with for D80, D80 (no N), and S94, while pure no-slip are used in the runs with S63, qDNS, and PA00. Small random perturbations are added to the surface forcing to excite instabilities and encourage the flow to become turbulent. Figure 2 shows the DNS output of the downslope velocity component, averaged over planes parallel to the surface, as a function of time and slope-normal height for a slope of $60^{\circ}$. The flow exhibits a characteristic oscillation in time with a period of $2 \pi /(N \sin \alpha)$, as has been observed in previous katabatic flow studies [13]. 
Table 1. List of abbreviations for different DNS/LES runs. The asterisk indicates that the mixing length scaling factor in Eq. 12 was not used.

\begin{tabular}{ll}
\hline Abbreviation Type (citation) \\
\hline DNS & DNS \\
fDNS & DNS (filtered to LES grid) \\
qDNS & LES (no closure) \\
S63 & LES [14] \\
D80 & LES [16] \\
D80 (no N) & LES [16]* \\
S94 & LES [22] \\
PA00 & LES [27][28] \\
\hline
\end{tabular}

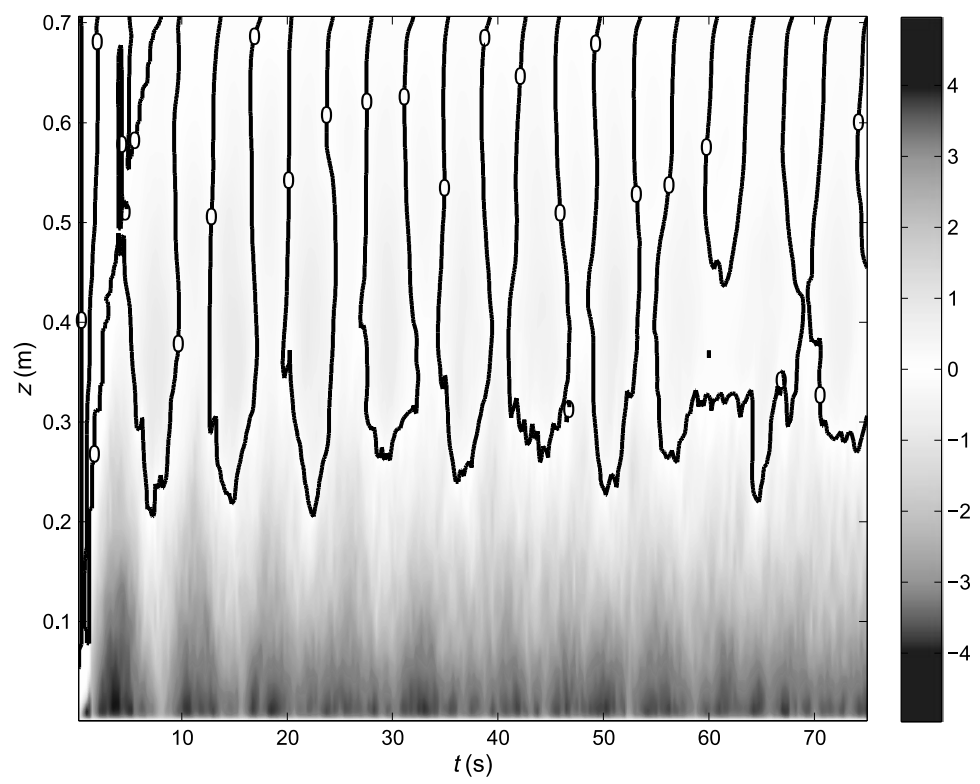

Fig. 2. DNS results of downslope velocity in a katabatic flow along a $60^{\circ}$ sloping surface. The bold contour demarcates the transition between positive and negative values of the along-slope component of velocity.

The two parameters that completely determine the flow in this case are $\alpha$ and $\left|F_{0}\right| v^{-1} N^{-2}$, where $F_{0}$ is the buoyancy flux imposed at the surface [13][29]. The characteristic length, velocity, and buoyancy scales of the flow in the present study are given, respectively, by

$$
l_{s}=\sqrt{\left|F_{0}\right| N^{-3}}, u_{s}=\sqrt{\left|F_{0}\right| N^{-1}}, b_{s}=\sqrt{\left|F_{0}\right| N} .
$$


Taking the governing parameters for the flow to be $F_{0}=-0.5 \mathrm{~m}^{2} \mathrm{~s}^{-3}, N=$ $1 \mathrm{~s}^{-1}$, and $v=10^{-4} \mathrm{~m}^{2} \mathrm{~s}^{-1}$, we obtain $\left|F_{0}\right| v^{-1} N^{-2}=5000$ with characteristic scales $l_{s}=0.71 \mathrm{~m}, u_{s}=0.71 \mathrm{~ms}^{-1}$, and $b_{s}=0.71 \mathrm{~ms}^{-2}$. Alternatively, we can choose other parameter values provided that $\left|F_{0}\right| / v N^{2}=5000$, which would result in exactly the same scaled flow solutions. For example, selecting values commonly found in atmospheric applications such as $N=10^{-2} \mathrm{~s}^{-1}, v=0.1 \mathrm{~m}^{2} \mathrm{~s}^{-1}$, and $F_{0}=$ $-0.05 \mathrm{~m}^{2} \mathrm{~s}^{-3}$, our characteristic scales become $l_{s 2}=220 \mathrm{~m}, u_{s 2}=2.2 \mathrm{~ms}^{-1}$, and $b_{s 2}=0.022 \mathrm{~ms}^{-2}$. Throughout the paper, we use the first set of scales to present results in a dimensional form.

\section{A Posteriori Testing}

A posteriori testing is commonly used to compare LES flow statistics to the those provided by a DNS of an identically forced flow. Since DNS typically has a higher resolution than LES, the DNS fields must be filtered down to the LES grid. To do this, a top-hat filter that has the width of the LES filter is applied. The effect of filtering the DNS output to the LES grid is shown in the top two panels of Fig. 3. It is seen that some of the smaller-scale features are lost through the filtering operation, but the overall visual characteristics of the flow do not change significantly. Since the filtered DNS output would be the best field LES could ever reproduce at a degraded resolution, it is taken as a reference flow field.

Normally, differences found in comparing an LES output to the filtered DNS results could potentially originate from different SGS models, numerical discretizations in space, time advancement schemes, or resolutions [4]. In our case, since each LES run is completed using identical numerical schemes, virtually all of the differences are expected to originate from the SGS models. In Fig. 3, we can see that near the surface, all tested SGS closures tend to organize the near-surface flow into largescale structures which are absent in the filtered DNS field. This could be due to the over-dissipative nature found in some of the schemes, but also may be an effect of the insufficient grid resolution near the surface. The level depicted in Fig. 3 is only the 8th point above the surface for LES, while it is the 32 nd point above the ground for DNS. It is likely the problems that LES has near bounding surfaces in moderate to high Reynolds number flows are still observed at this level above the lower boundary. The dynamic closure and the qDNS share the most visual similarities with the filtered DNS output. This may be due to the the schemes being under-dissipative, which is briefly discussed below.

The one-dimensional velocity spectra shown in Fig. 4 demonstrate that most of the SGS closures produce the desired general spectral characteristics. The velocity spectra are calculated according to [30] and are averaged in time over at least six oscillations (see Fig. 2). This smooths the spectra making them easier to interpret. Near the surface, LES spectra obtained with S63 and S94 schemes are almost identical to the fDNS spectrum. The one spectrum that does not exhibit reasonable behavior is from the qDNS, which follows the $-5 / 3$ line at exceedingly high wavenumbers. In the plot with their dissipation spectra, $D_{11}(k)=2 v k^{2} E_{11}(k)$, turbulence in the qDNS is 

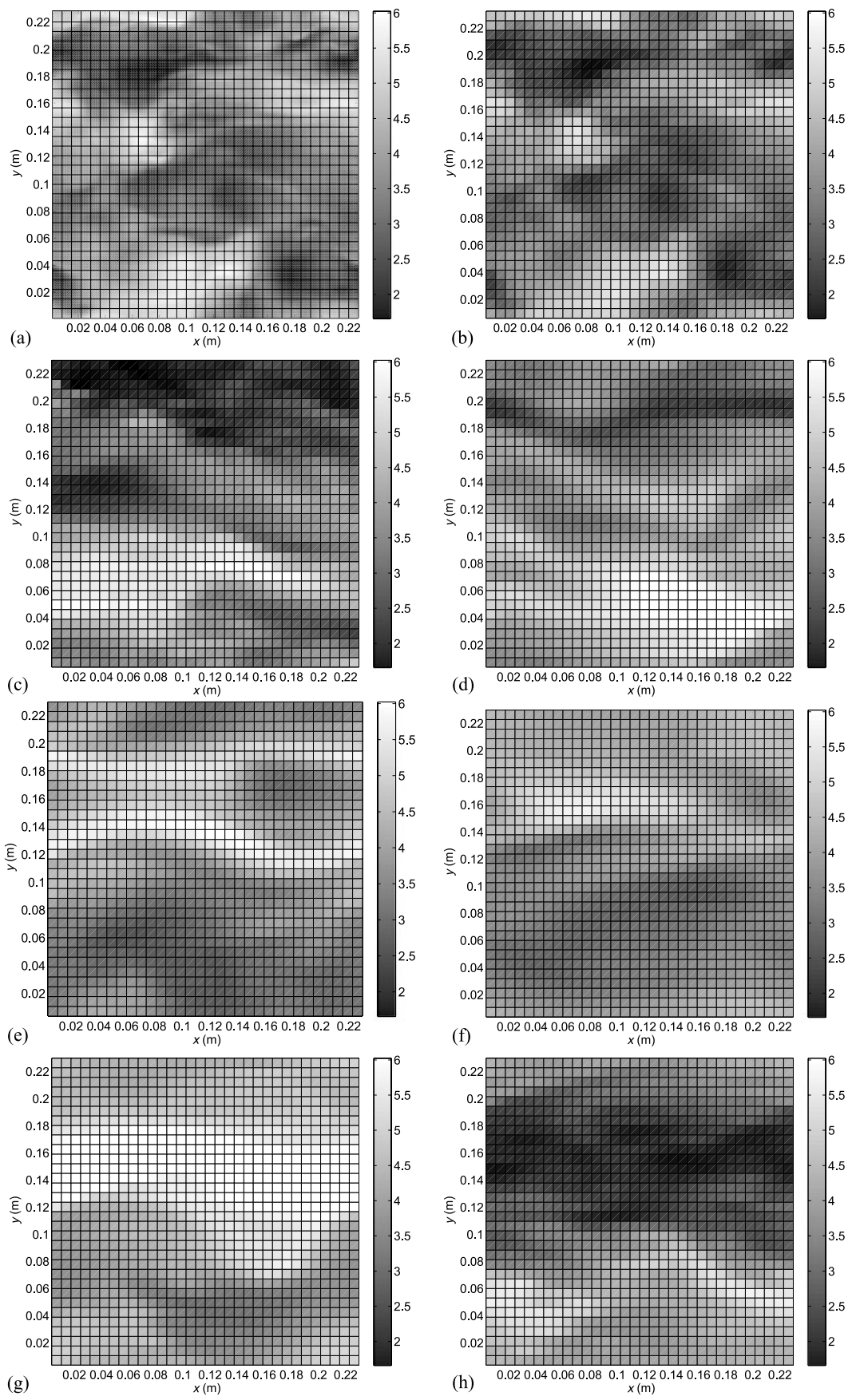

Fig. 3. Horizontal contour plot of downslope velocity on a plane parallel to the sloping surface. Each panel starting at the top left and going to the right and down, shows a) the output from DNS, b) the DNS output filtered to the LES grid, c) qDNS, d) S63, e) D80, f) D80 (no N), g) $\mathrm{S} 94$, and $\mathrm{h}$ ) PA00. In each plot, the LES grid spacing is illustrated by the thin black lines. 
found to be dissipating improperly. Most of the energy in this spectrum dissipates at wavenumbers very close to the Kolmogorov scale. All of the SGS closure schemes show reasonable spectral dissipation properties, as most of the dissipation occurs at wavenumbers much smaller than the Kolmogorov scale. The discrepancies between the different SGS closures become smaller with height, as the LES is able to resolve the motions at these levels. However, the D80 TKE-based schemes appear to be slightly over-dissipative, even at substantial distances from the slope. For katabatic flows, the S63 scheme performance appears to be reasonable, at least once turbulence has already developed. As has been previously reported, the dynamic model is under-dissipative close to the surface [27], but performs reasonably well in regions away from the surface.
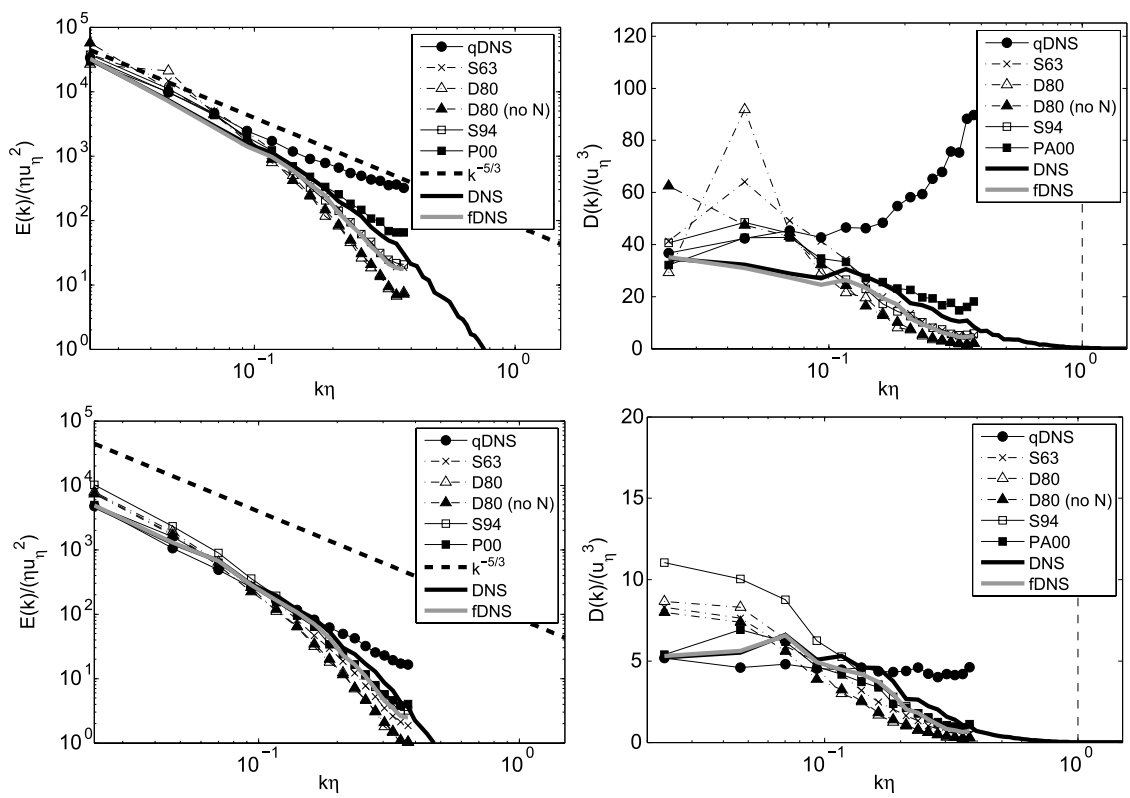

Fig. 4. Time-averaged normalized $u_{1}$ spectra at DNS level 32 (LES level 8) (top, left) and their respective dissipation spectra (top,right). The same is shown at level 160 in the bottom two panels.

From Fig. 5, we conclude that LES is roughly capable of reproducing the correct flow in terms of mean velocity and buoyancy which are obtained by averaging over planes parallel to the surface and over six oscillations in time. Not surprisingly, the LES performance is worse for higher order statistics, as is seen for the total turbulent fluxes and the variances, especially near the surface. When comparing the ratios of the total contribution of each velocity variance to the to total velocity component variance, we can see that the fDNS exhibits $\left\langle\widetilde{u_{1}^{\prime} u_{1}^{\prime}}\right\rangle$ that is about 3 times larger than $\left\langle\widetilde{u_{3}^{\prime} u_{3}^{\prime}}\right\rangle$, where the angle brackets here denote the combined spatial (over planes paral- 

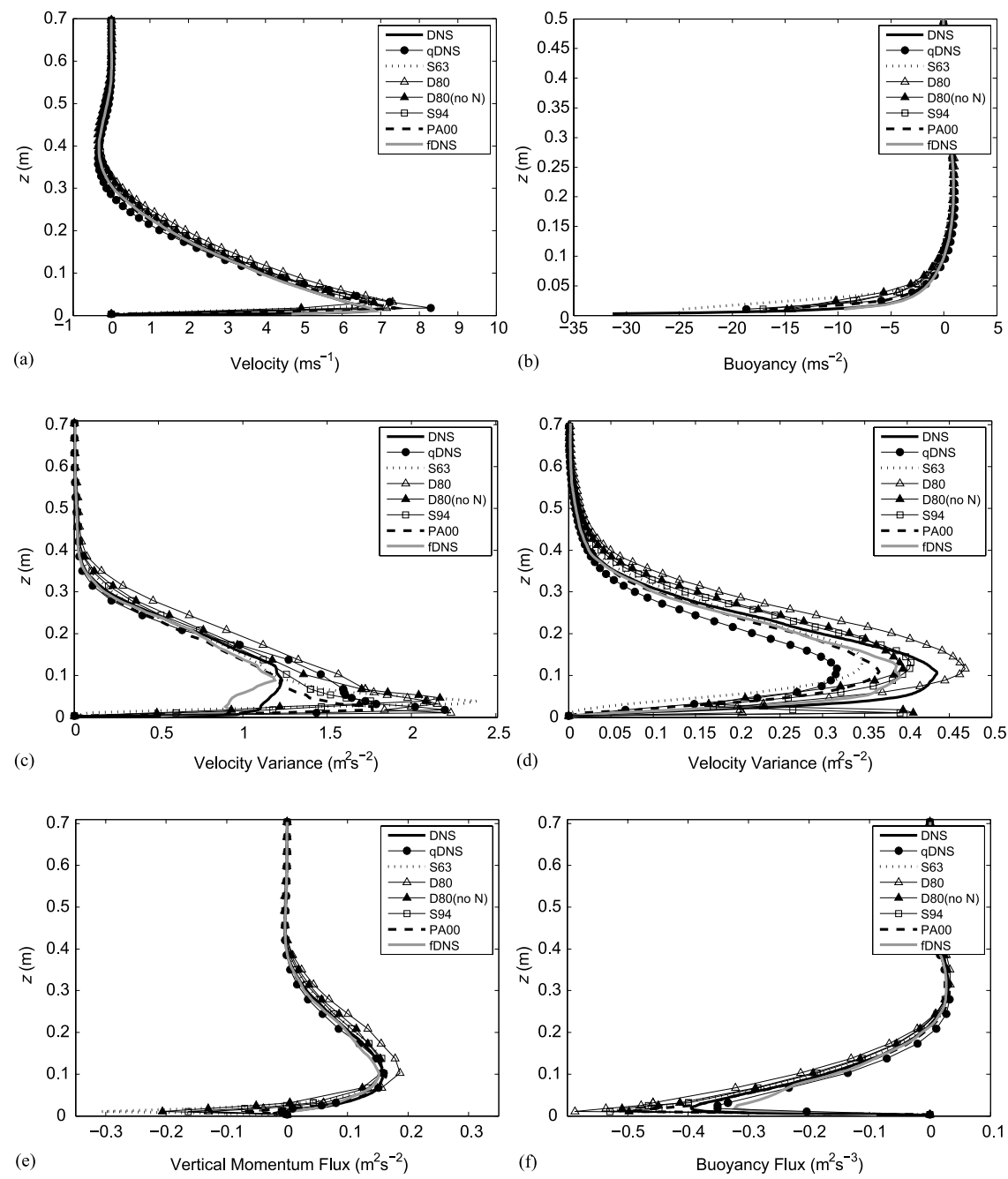

Fig. 5. Temporal and planar-averaged profiles $(\langle\ldots\rangle)$ of a) $\left\langle\tilde{u_{1}}\right\rangle$, b) $\langle\tilde{b}\rangle$, c) $\left\langle\widetilde{u_{1}^{\prime} u_{1}^{\prime}}\right\rangle$, d) $\left\langle\widetilde{u_{3}^{\prime} u_{3}^{\prime}}\right\rangle$, e) $\left\langle\widehat{\left\langle u_{1}^{\prime} u_{3}^{\prime}\right.}\right\rangle$, and f) $\left\langle\widetilde{\left\langle u_{3}^{\prime} b^{\prime}\right.}\right\rangle$ as a function of $z$.

lel to the slope) and temporal (over several flow oscillations) averaging. Meanwhile, LES tends to have larger ratios of variance components, with $\left\langle\widehat{\left\langle u_{1}^{\prime} u_{1}^{\prime}\right.}\right\rangle$ being about a factor of 4.5 - 10 times larger than $\left\langle\widetilde{u_{3}^{\prime} u_{3}^{\prime}}\right\rangle$. Near the surface, the TKE-based SGS closures generate a pronounced spike in vertical velocity variance. An analysis of the subgrid TKE budget (not shown) reveals that the near-surface shear production associated with the katabatic jet is responsible for the spike. Some of the SGS models tested (especially the S63 model) also tend to over-predict the slightly negative verti- 
cal momentum flux near the surface that is observed in the DNS output. Interestingly, for the buoyancy related fields, the LES with no SGS model (qDNS) outperformed some LES with SGS formulations. It should be noted that the correction of the Sullivan [22] scheme for scalars implemented in [23] was not tested.

\section{Conclusions}

Overall, the performance of the basic SGS closures employed by LES for atmospheric applications is acceptable for mean fields, but degrades noticeably when it comes to higher-order statistics (particularly buoyancy fluxes). Near the ground, where the SGS contributions compose the largest portion of the total fluxes and variances, the different closures produce highly variable results. The over-production of subgrid TKE by the shear close to the surface causes errors in the models based on [16] and especially affect the vertical velocity variance. Also, many of the tested schemes drastically overestimate the negative momentum flux near the ground. A majority of the tested schemes are able to capture the anisotropy of the flow in the along-slope direction, though this feature of the flow is also slightly overestimated by the LES.

With respect to velocity spectra, the [22] and [14] schemes perform the best near the ground, while the dynamic and two-part models perform better in turbulent regions of the flow away from the surface. Even though the solution produced by the qDNS remains stable, it exhibits a build-up of energy as well as unrealistic dissipation at high wavelengths. The schemes based on [16] are found to be over-dissipative, and flow statistics slightly improved when the mixing length stratification adjustment after [16] is not used.

The extension of testing toward other SGS models will constitute the scope of future studies. Updating the time advancement schemes in both DNS and LES is also planned. The influence of the varying slope angle on the performance of the SGS models in LES of katabatic flows should be studied as well.

\section{References}

1. Mason PJ (1994) Bound-Layer Meteor 120:1-26

2. Mahrt L (1998) Theoret Comput Fluid Dyn 11:263-279

3. Beare RJ, MacVean MK, Holtslag AAM, Cuxart J, Esau I, Golaz JC, Jiménez MA, Khairoutdinov M, Kosović B, Lewellen D, Lund TS, Lundquist JK, McCabe A, Moene AF, Noh Y, Raasch S, and Sullivan P (2006) Bound-Layer Meteor 118:247-272

4. Geurts B (2004) Elements of direct and large-eddy simulation. R. T. Edwards, Inc., Philadelphia, PA

5. Vreman B, Geurts B, and Kuerten H (1997) J Fluid Mech 339:357-390

6. Prandtl L (1942) Führer durch die Strömungslehre. Vieweg \& Sohn, Braunschweig

7. Denby B (1999) Bound-Layer Meteor 92:67-100

8. Pope SB (2000) Turbulent flows. Cambridge University Press, Cambridge

9. Schumann U (1990) Quart J Roy Meteor Soc 116:637-670 
10. Skyllingstad ED (2003) Bound-Layer Meteor 106:217-243

11. Shapiro A and Fedorovich E (2004) Int J Heat and Mass Transfer 47:4911-4927

12. Burkholder BA, Shapiro A, Fedorovich E (2009) Acta Geophysica (in press, DOI: 10.2478/s11600-009-0025-6)

13. Fedorovich E and Shapiro A (2009) Acta Geophysica (in press, DOI: 10.2478/s11600009-0027-4)

14. Smagorinsky J (1963) Mon Wea Rev 91:99-164

15. Lilly DK (1967) Proc IBM Sci Computing Symp Environmental Sci 320-1951:195-210

16. Deardorff JW (1980) Bound-Layer Meteor 18:495-527

17. Piomelli U and Chasnov JR (1996) Large-eddy simulations: theory and applications. In: Henningson D, Hallbäck, Alfreddson H, and Johansson A (eds) Transition and turbulence modelling. Kluwer Academic Publishers, Dordrecht

18. Leslie DC and Quarini GL (1979) J Fluid Mech 91:65-91

19. Mason PJ and Thomson DJ (1992) J Fluid Mech 242:51-78

20. Brown AR, Derbyshire SH, Mason PJ (1994) Quart J Roy Meteorol Soc 120:1485-1512

21. Kosović B (1997) J Fluid Mech 336:151-182

22. Sullivan PP, McWilliams JC, Moeng C (1994) Bound-Layer Meteor 71:247-276

23. Saiki EM, Moeng C, and Sullivan PP (2000) Bound-Layer Meteor 95:1-30

24. Germano M, Piomelli U, Moin P, Cabot WH (1991) Phys Fluids A 7:1760-1771

25. Lilly DK (1992) Phys Fluids A 4:633-635

26. Stoll R and Porté-Agel F (2008) Bound-Layer Meteor 126:1-28

27. Porté-Agel F, Meneveau C, Parlange MB (2000) J Fluid Mech 415:261-284

28. Porté-Agel (2004) Bound-Layer Meteor 112:81-105

29. Fedorovich E and Shapiro A (2009) J Fluid Mech (in press)

30. Kaiser R and Fedorovich E (1998) J Atmo Sci 55:580-594 